\title{
MOA-2010-BLG-328Lb: A SUB-NEPTUNE ORBITING VERY LATE M DWARF?
}

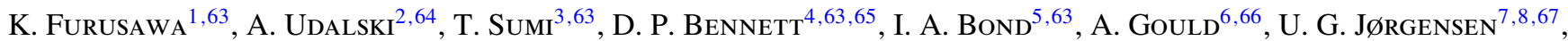 \\ C. Snodgrass ${ }^{9,10,67,68}$, D. Dominis Prester ${ }^{11,65}$, M. D. Albrow ${ }^{12,65}$, \\ AND
}

F. Abe ${ }^{1}$, C. S. Botzler ${ }^{13}$, P. Chote ${ }^{14}$, M. Freeman ${ }^{13}$, A. Fukui ${ }^{15}$, P. Harris ${ }^{14}$, Y. Itow ${ }^{1}$, C. H. Ling ${ }^{5}$, K. Masuda ${ }^{1}$, Y. Matsubara ${ }^{1}$, N. Miyake ${ }^{1}$, Y. Muraki ${ }^{1}$, K. Ohnishi ${ }^{16}$, N. J. Rattenbury ${ }^{13}$, To. Saito ${ }^{17}$, D. J. Sullivan ${ }^{14}$, D. Suzuki ${ }^{3}$, W. L. Sweatman ${ }^{5}$, P. J. Tristram ${ }^{18}$, K. WADA ${ }^{3}$, P. C. M. Yock ${ }^{13}$

(The MOA Collaboration)

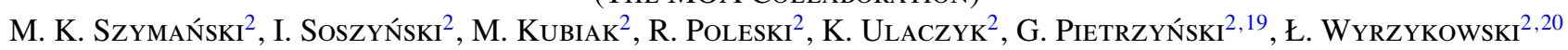
(The OGLE COLlaboration)

J.-Y. Choi ${ }^{21}$, G. W. Christie ${ }^{22}$, D. L. DePoy ${ }^{23}$, Subo Dong ${ }^{24}$, J. Drummond ${ }^{25}$, B. S. Gaudi ${ }^{6}$, C. Han ${ }^{21}$, L.-W. Hung ${ }^{6,26}$,

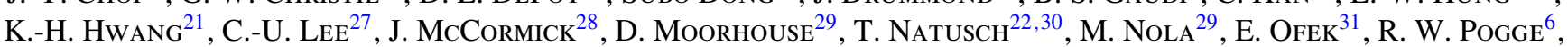
I.-G. Shin ${ }^{21}$, J. SKOWRON ${ }^{6}$, G. ThORnLEY ${ }^{29}$, J. C. YeE

(THE $\mu$ FUN COLlaboration)

K. A. Alsubai ${ }^{32}$, V. Bozza ${ }^{33,34}$, P. Browne ${ }^{35,68}$, M. J. Burgdorf ${ }^{36}$, S. Calchi Novati ${ }^{33,37}$, P. Dodds ${ }^{35}$, M. Dominik ${ }^{35,65,68,69}$,

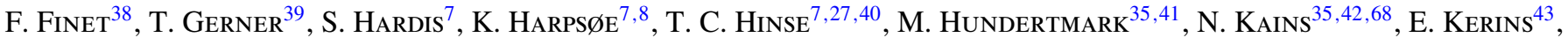

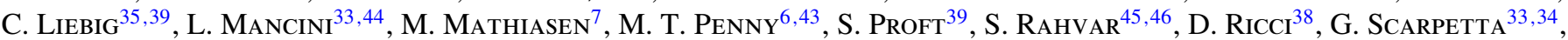
S. Schäfer ${ }^{41}$, F. Schönebeck ${ }^{39}$, J. Southworth ${ }^{47}$, J. Surdej ${ }^{41}$, J. WAMbSGanss $^{39}$

(The MiNDSTEp CONSORTIUM)

\section{R. A. Street $^{48}$, D. M. Bramich ${ }^{42}$, I. A. Steele ${ }^{49}$, Y. Tsapras ${ }^{48,50}$}

(The Robonet Collaboration)

K. Horne ${ }^{35,68}$, J. Donatowicz ${ }^{51}$, K. C. SAhu ${ }^{52,67}$, E. Bachelet ${ }^{53}$, V. Batista ${ }^{6,54}$, T. G. Beatty ${ }^{6}$, J.-P. Beaulieu ${ }^{54}$, C. S. BennetT ${ }^{55}$, C. Black ${ }^{56}$, R. Bowens-Rubin ${ }^{57}$, S. Brillant ${ }^{10}$, J. A. R. Caldwell ${ }^{58}$, A. Cassan ${ }^{54}$, A. A. Cole $^{56}$, E. Corrales $^{54}$, C. Coutures ${ }^{54}$, S. Dieters ${ }^{56}$, P. Fouqué ${ }^{53}$, J. Greenhill ${ }^{56}$, C. B. Henderson ${ }^{6}$, D. Kubas $^{10,54}$, J.-B. Marquette ${ }^{54}$, R. Martin ${ }^{59}$, J. W. Menzies ${ }^{60}$, B. Shappee ${ }^{6}$, A. Williams ${ }^{59}$, D. Wouters ${ }^{61}$, J. VAN SADERS ${ }^{6}$, R. ZELLEM ${ }^{62}$, M. ZuB ${ }^{38}$

(The PLANET COLlaboration)

${ }^{1}$ Solar-Terrestrial Environment Laboratory, Nagoya University, Nagoya 464-8601, Japan; furusawa @ stelab.nagoya-u.ac.jp

${ }^{2}$ Warsaw University Observatory, Al. Ujazdowskie 4, 00-478 Warszawa, Poland

${ }^{3}$ Department of Earth and Space Science, Graduate School of Science, Osaka University, 1-1 Machikaneyama-cho, Toyonaka, Osaka 560-0043, Japan

${ }^{4}$ Department of Physics, 225 Nieuwland Science Hall, University of Notre Dame, Notre Dame, IN 46556, USA

${ }^{5}$ Institute for Information and Mathematical Sciences, Massey University, Private Bag 102-904, Auckland 1330, New Zealand

${ }^{6}$ Department of Astronomy, Ohio State University, 140 West 18th Avenue, Columbus, OH 43210, USA

${ }^{7}$ Niels Bohr Institutet, Københavns Universitet, Juliane Maries Vej 30, 2100 Copenhagen, Denmark

${ }^{8}$ Centre for Star and Planet Formation, Geological Museum, Øster Voldgade 5, 1350 Copenhagen, Denmark

${ }^{9}$ Max Planck Institute for Solar System Research, Max-Planck-Str. 2, D-37191 Katlenburg-Lindau, Germany

${ }^{10}$ European Southern Observatory, Casilla 19001, Vitacura 19, Santiago, Chile

${ }^{11}$ Department of Physics, University of Rijeka, Omladinska 14, 51000 Rijeka, Croatia

12 Department of Physics and Astronomy, University of Canterbury, Private Bag 4800, Christchurch 8020, New Zealand

${ }^{13}$ Department of Physics, University of Auckland, Private Bag 92-019, Auckland 1001, New Zealand

${ }^{14}$ School of Chemical and Physical Sciences, Victoria University, Wellington, New Zealand

${ }^{15}$ Okayama Astrophysical Observatory, National Astronomical Observatory of Japan, 3037-5 Honjo, Kamogata, Asakuchi, Okayama 719-0232, Japan

${ }_{16}$ Nagano National College of Technology, Nagano 381-8550, Japan

${ }^{17}$ Tokyo Metropolitan College of Aeronautics, Tokyo 116-8523, Japan

${ }^{18}$ Mt. John University Observatory, P.O. Box 56, Lake Tekapo 8770, New Zealand

${ }^{19}$ Departamento de Astronomía, Universidad de Concepción, Casilla 160-C, Concepción, Chile

${ }^{20}$ Institute of Astronomy, University of Cambridge, Madingley Road, Cambridge CB3 OHA, UK

${ }^{21}$ Department of Physics, Chungbuk National University, 410 Seongbong-Rho, Hungduk-Gu, Chongju 371-763, Korea

22 Auckland Observatory, P.O. Box 24-180, Auckland, New Zealand

${ }^{23}$ Department of Physics, Texas A\&M University, 4242 TAMU, College Station, TX 77843-4242, USA

${ }^{24}$ Institute for Advanced Study, Einstein Drive, Princeton, NJ 08540, USA

${ }^{25}$ Possum Observatory, Patutahi, New Zealand

${ }^{26}$ Department of Physics \& Astronomy, University of California Los Angeles, Los Angeles, CA 90095, USA; liweih@astro.ucla.edu

${ }^{27}$ Korea Astronomy and Space Science Institute, 776 Daedukdae-ro, Yuseong-gu 305-348 Daejeon, Korea

${ }^{28}$ Farm Cove Observatory, 2/24 Rapallo Place, Pakuranga, Auckland 1706, New Zealand

${ }^{29}$ Kumeu Observatory, Kumeu, New Zealand

${ }^{30}$ Institute for Radiophysics and Space Research, AUT University, Auckland, New Zealand; tim.natusch@aut.ac.nz

${ }^{31}$ Wise Observatory, Tel Aviv University, Ramat Aviv, Tel Aviv 69978, Israel

${ }^{32}$ Qatar Foundation, P.O. Box 5825, Doha, Qatar

${ }^{33}$ Dipartimento di Fisica E. R. Caianiello, Università degli Studi di Salerno, Via Ponte Don Melillo, I-84084 Fisciano, Italy

${ }^{34}$ INFN, Sezione di Napoli, Italy

${ }^{35}$ School of Physics \& Astronomy, SUPA, University of St Andrews, North Haugh, St Andrews KY16 9SS, UK

${ }^{36}$ HE Space Operations, Flughafenallee 26, D-28199 Bremen, Germany

${ }^{37}$ Istituto Internazionale per gli Alti Studi Scientifici (IIASS), Vietri Sul Mare (SA), Italy

${ }^{38}$ Institut d'Astrophysique et de Géophysique, Allée du 6 Août 17, Sart Tilman, Bât. B5c, 4000 Liège, Belgium 


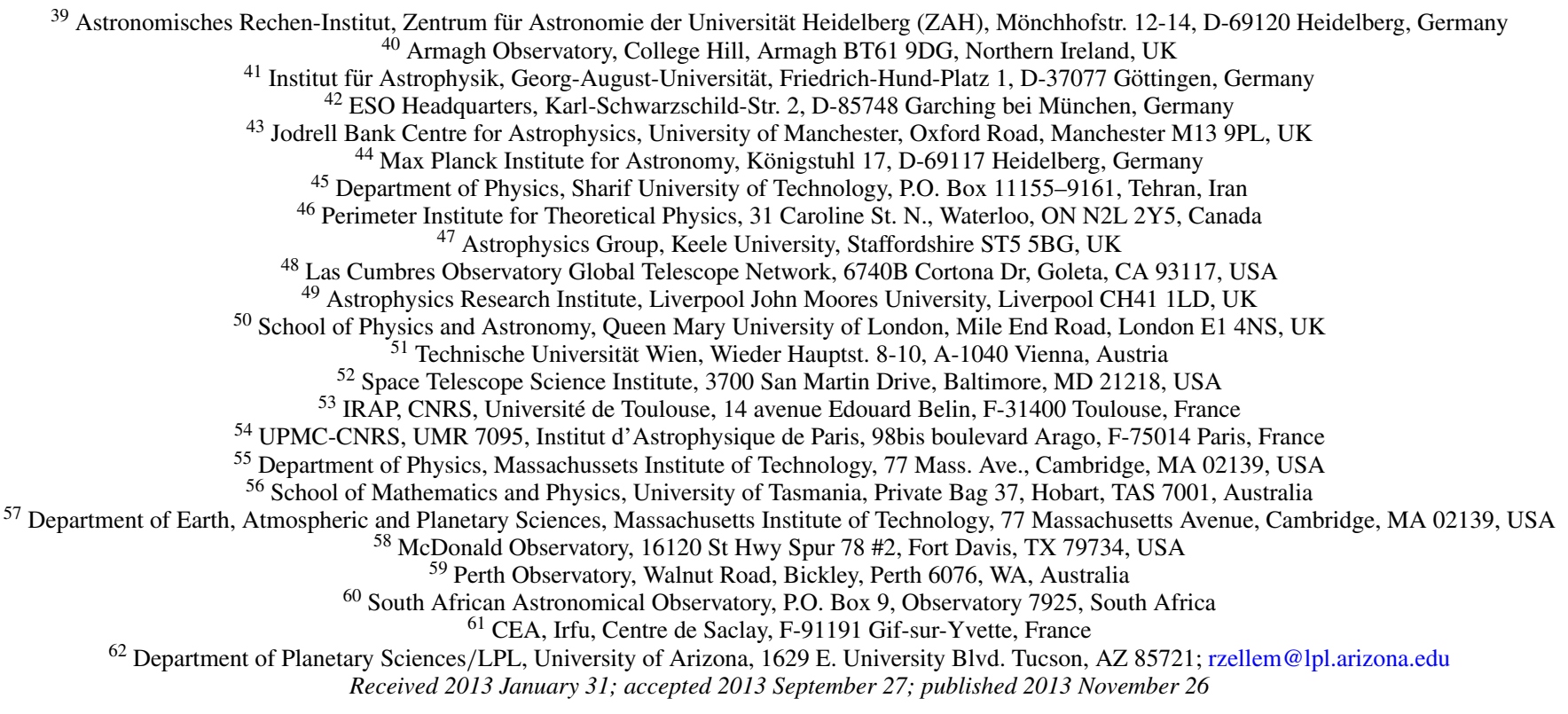

\begin{abstract}
We analyze the planetary microlensing event MOA-2010-BLG-328. The best fit yields host and planetary masses of $M_{\mathrm{h}}=0.11 \pm 0.01 M_{\odot}$ and $M_{\mathrm{p}}=9.2 \pm 2.2 M_{\oplus}$, corresponding to a very late $\mathrm{M}$ dwarf and sub-Neptune-mass planet, respectively. The system lies at $D_{\mathrm{L}}=0.81 \pm 0.10 \mathrm{kpc}$ with projected separation $r_{\perp}=0.92 \pm 0.16 \mathrm{AU}$. Because of the host's a priori unlikely close distance, as well as the unusual nature of the system, we consider the possibility that the microlens parallax signal, which determines the host mass and distance, is actually due to xallarap (source orbital motion) that is being misinterpreted as parallax. We show a result that favors the parallax solution, even given its close host distance. We show that future high-resolution astrometric measurements could decisively resolve the remaining ambiguity of these solutions.
\end{abstract}

Key words: gravitational lensing: micro - planetary systems

\section{INTRODUCTION}

To date, more than 800 exoplanets have been discovered via several different methods. Most of the exoplanets have been discovered with the radial velocity (Lovis \& Fischer 2011) and transit methods (Winn 2011). These methods are most sensitive to planets in very close orbits and, as a result, our understanding of the properties of exoplanetary systems is dominated by planets in close orbits. While the number of exoplanet discoveries by microlensing is relatively small (18 discoveries to date; Bond et al. 2004; Bachelet et al. 2012), microlensing is sensitive to planets beyond the "snow line" at $\sim 2.7 \mathrm{AU}\left(M / M_{\odot}\right)$ (Kennedy \& Kenyon 2008), where $M$ is the mass of the host star. This region beyond the "snow line" is thought to be the dominant exoplanet birthplace and microlensing is able to find planets down to an Earth mass (Bennett \& Rhie 1996) in this region. Microlensing does not depend on the detection of any light from the exoplanet host stars, so planets orbiting faint hosts, like brown dwarfs and

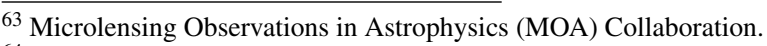

${ }^{64}$ Optical Gravitational Lensing Experiment (OGLE).

${ }^{65}$ Probing Lensing Anomalies Network (PLANET) Collaboration.

${ }^{66}$ Microlensing Follow Up Network ( $\mu$ FUN) Collaboration.

${ }^{67}$ Microlensing Network for Detection of Small Terrestrial Exoplanets (MiNDSTEp) Consortium.

${ }^{68}$ RoboNet Collaboration.

${ }^{69}$ Royal Society University Research Fellow.
}

M dwarfs (Udalski et al. 2005; Dong et al. 2009; Bennett et al. 2008; Kubas et al. 2012; Batista et al. 2011), can be detected.

Microlensing is one of several methods that has contributed to our statistical understanding of the exoplanet distribution. In other methods, Cumming et al. (2008) analyzed 8 yr of radial velocity measurements to constraint the frequency of Jupitermass planets (0.3-10 $\left.M_{\text {Jupiter }}\right)$ with orbital periods of less than 2000 days and found that less than $10.5 \%$ of the stars in their sample had such planets. Wittenmyer et al. (2011) used a $12 \mathrm{yr}$ radial velocity sample to search for Jupiter analogs and found that between $3.3 \%$ and $37.2 \%$ of the stars in their sample had a planet with such a mass. When it comes to the transit method, Howard et al. (2012) reported the distribution of planets as a function of planet radius, orbital period, and stellar effective temperature for orbital periods less than 50 days around solartype stars. They measured an occurrence of $0.165 \pm 0.008$ planets per star for planets with radii $2-32 R_{\oplus}$. Microlensing has already demonstrated the ability to find both Jupiter- and Saturn-analog planets with the discovery of the Jupiter/Saturn analog system, OGLE-2006-BLG-109Lb,c (Gaudi et al. 2008; Bennett et al. 2010). There have been several recent papers that have looked at the statistical implications of the microlensing exoplanet discoveries. Sumi et al. (2010) determined the slope of the exoplanet mass function beyond the snow line and found that the cold Neptunes are $\sim 7$ times more common than Jupiters. (The 95\% confidence level limit is more than three times more common.) Gould et al. (2010) used six microlensing discoveries to show that low-mass gas giant planets are quite 
common beyond the snow line of low-mass stars at a level that is consistent with an extrapolation of the Cumming et al. (2008) radial velocity results. Most recently, Cassan et al. (2012) estimated the fraction of bound planets at separations of 0.5-10 AU with a somewhat larger microlensing sample. They found that $17_{-9}^{+6} \%$ of stars host Jupiter-mass planets, while $52_{-29}^{+22} \%$ and $62_{-37}^{+35} \%$ of stars host Neptune-mass planets $\left(10-30 M_{\oplus}\right)$ and super-Earths $\left(5-10 M_{\oplus}\right)$, respectively.

In this paper, we present an analysis of the planetary microlensing event MOA-2010-BLG-328. Section 2 describes the observation of the event and the light curve modeling is presented in Section 3. The light curve shows evidence of orbital motion of either the Earth, known as microlensing parallax (Smith et al. 2003), or of the source stars, which is often referred to as the xallarap effect. These two possibilities have very different implications for the properties of the host star and its planet. However, we need the angular radius of the source star to work out the implications for the star plus planet lens system, so we determine the source star angular radius in Section 4. In Section 5, we present the implications for the properties of the host star and its planet for both the parallax and xallarap solutions. While the data do prefer the parallax solution, the xallarap model is not completely excluded. In Section 6, we describe how future follow-up observations can distinguish between these two solutions and we give our conclusions.

\section{OBSERVATIONS}

Several groups search for exoplanets using the microlensing method, using two different observing modes: wide field-ofview (FOV) surveys and narrow FOV follow-up observations. The microlensing surveys active in 2010 were the Microlensing Observations in Astrophysics (MOA; Bond et al. 2001; Sumi et al. 2003) group and the Optical Gravitational Lensing Experiment (OGLE; Udalski 2003). MOA uses the $1.8 \mathrm{~m}$ MOA-II telescope equipped with the $10 \mathrm{k} \times 8 \mathrm{k}$ pixel $\mathrm{CCD}$ camera MOA-cam3 (Sako et al. 2008) with a $2.2 \mathrm{deg}^{2}$ FOV to monitor $\sim 44 \mathrm{deg}^{2}$ of the Galactic bulge with a cadence of one observation of each field every 15-95 minutes depending on the field. MOA identifies microlensing events in real time (Bond et al. 2001) and announced 607 microlensing alerts in 2010. In 2010, the OGLE collaboration initiated the OGLE-IV survey, after upgrading their CCD camera from the OGLE-III system, which operated from 2001-2009. OGLE observations are conducted with $1.3 \mathrm{~m}$ Warsaw telescope at Las Campanas Observatory, Chile. The OGLE-IV survey employs a $1.4 \mathrm{deg}^{2}, 256$ megapixel, 32 chip CCD mosaic camera to survey an even larger area of the Galactic bulge at cadences ranging from one observation every 20 minutes to fewer than one observation per day. The OGLE real-time event detection system, known as the early warning system (EWS; Udalski 2003), was not operational in 2010.

The follow-up groups employ narrow FOV telescopes spread across the world (mostly in the Southern hemisphere) for highcadence photometric monitoring of a subset of microlensing events found by the survey groups. Generally, the events observed by the follow-up groups are events with a high planet detection sensitivity (Griest \& Safizadeh 1998; Horne et al. 2009) or events in which a candidate planetary signal has been seen. Follow-up groups include the Microlensing Follow-Up Network ( $\mu$ FUN; Gould et al. 2006), the Microlensing Network for the Detection of Small Terrestrial Exoplanets (MiNDSTEp; Dominik et al. 2010), RoboNet (Tsapras et al. 2009), and the Probing Lensing Anomalies NETwork (PLANET; Beaulieu et al. 2006). Because the planetary deviations are short, with durations ranging from a few hours to a few days, high-cadence observations from observatories widely spaced in longitude are needed to provide good sampling.

The microlensing event MOA-2010-BLG-328 (R.A., decl.) $(\mathrm{J} 2000)=\left(17^{\mathrm{h}} 57^{\mathrm{m}} 59^{\mathrm{s}} .12,-30^{\circ} 42^{\prime} 54^{\prime \prime} .63\right)[(l, b)=(-0.16$, $-3.21)$ ] was detected and alerted by MOA on 2010 June 16 (HJD' $\equiv$ HJD - $2450000 \sim 5363$ ). The MOA observer noticed a few data points at $\mathrm{HJD}^{\prime} \sim 5402$ that were above the prediction of the single lens light curve model, but waited for the next observations, three days later, for the significance of the deviation to reach the threshold to issue an anomaly alert. This anomaly alert was issued to the other microlensing groups at UT 11:30 27 July ( HJD $\left.^{\prime} \sim 5405\right)$. One day later, MOA circulated a preliminary planetary model and, shortly thereafter, observations were begun by the follow-up groups. Follow-up data were obtained from the $\mu$ FUN, PLANET, MiNDSTEp, and RoboNet groups. $\mu$ FUN obtained data from the CTIO $1.3 \mathrm{~m}$ telescope in Chile in the $I, V$, and $H$ bands, the Palomar Observatory $1.5 \mathrm{~m}$ telescope in the United States in the $I$ band, and the Farm Cove Observatory $0.36 \mathrm{~m}$ telescope in New Zealand in the unfiltered pass band. $\mu$ FUN also obtained data from Auckland Observatory, Kumeu Observatory, and Possum Observatory, all in New Zealand; unfortunately, they obtained only one night of observations, so these data are not used in the analysis. The datasets from PLANET consist of $V$ - and $I$-band data from the SAAO $1.0 \mathrm{~m}$ telescope in South Africa and $I$-band data from the Canopus Observatory $1.0 \mathrm{~m}$ telescope in Australia. RoboNet provided data from the Faulkes Telescope North $2.0 \mathrm{~m}$ telescope in Hawaii in the $V$ and $I$ bands, the Faulkes Telescope South $2.0 \mathrm{~m}$ telescope in Australia in the $I$ band, and the Liverpool Telescope $2.0 \mathrm{~m}$ telescope in the Canary Islands in the $I$ band. The MiNDSTEp group provided data from the Danish $1.54 \mathrm{~m}$ telescope at the European Southern Observatory, La Silla in Chile in the I band. MOA's observations were done in the wide MOA-red band, which is approximately equivalent to $R+I$, and the observations during the main peak of the planetary deviation were taken at a cadence of about one image every 10 minutes, or four to five times higher than the normal observing cadence due to a high magnification event in the same field and the detection of the anomaly in this event. Due to poor weather at the Mt. John University Observatory, where the MOA telescope is located, the planetary signal was recognized and announced after it was already nearing the second peak, so the light curve coverage from the follow-up groups is poor. Fortunately, much of the early part of the planetary deviation was monitored by the OGLE-IV survey (in the $I$ band), and so we have good coverage of most of the planetary deviation from the MOA and OGLE surveys.

Most of the photometry was done by the standard difference imaging photometry method for each group. The MOA data were reduced with the MOA Difference Image Analysis (DIA) pipeline (Bond et al. 2001), and the OGLE data were reduced with the OGLE DIA photometry pipeline (Udalski 2003). The photometry of $\mu$ FUN and PLANET was performed with the PLANET group's PYSYS (Albrow et al. 2009) difference imaging code. The RoboNet data were reduced with DanDIA (Bramich 2008) and the MiNDSTEp data were reduced with DIAPL (Wozniak 2000). The CTIO $V$ - and $I$-band data were also reduced with DoPHOT (Schechter et al. 1993) in order to get photometry of the lensed source on the same scale as photometry of the non-variable bright stars in the frame. Since there were only two observations from the Faulkes Telescope South, we 
Table 1

The Dataset Used for the Modeling

\begin{tabular}{lcr}
\hline \hline Observatory & Filter & $N_{\text {data }}$ \\
\hline MOA & MOA-red & 3654 \\
OGLE & $I$ & 1436 \\
CTIO & $I$ & 118 \\
& $V$ & 10 \\
Palomar & $I$ & 26 \\
Farm Cove & Unfiltered & 44 \\
SAAO & $I$ & 133 \\
& $V$ & 6 \\
Canopus & $I$ & 37 \\
Faulkes North & $I$ & 62 \\
& $V$ & 4 \\
Liverpool & $I$ & 37 \\
Danish & $I$ & 131 \\
\hline
\end{tabular}

have not included these data in our modeling. Finally, we did not use MOA data from before 2009, because there appeared to be some systematic errors in the early baseline observations. The data sets used for the modeling are summarized in Table 1. The error bars provided by these photometry codes are generally good estimates of the relative error bars for the different data points, but they often provide only a rough estimate of the absolute uncertainty for each photometric measurement. Therefore, we follow the standard practices of renormalizing the error bars to give $\chi^{2} /$ degrees of freedom (dof) $\sim 1$ for each dataset once a reasonable model has been found. In this case, we have used the best parallax plus orbital model (see Section 3.2) for this error bar renormalization. This procedure ensures that the error bars for the microlensing fit parameters are calculated correctly. We carefully examined the properties of the residual distribution weighted by the normalized errors. We confirmed that it is well represented by the Gaussian distribution with a standard deviation of close to unity, $\sigma=0.94$, where the Kolmogorov-Smirnov probability is $4.8 \%$ and $6.9 \%$ for the unconstrained Xallarap model and the Parallax+orbital model, respectively (see the next section). The best-fit sigma of $\sigma=0.94$ is slightly smaller than unity due to compensating for the excess points in the tails of the residual distribution. The number of these excess points that are more than $3 \sigma$ deviant is not so large, $\sim 0.9 \%$, compared with the formally expected fraction of $0.27 \%$. Furthermore, they are sparsely distributed all over the light curve, i.e., they are not clustered at any particular place. We also found that they are not correlated with the seeing. There is only a weak correlation with airmass, of $\sim 0.1 \sigma$ per airmass, which is too small to explain the excess tails. So, we concluded that it is unlikely that they bias our result significantly. Thus, the effect of this small deviation from a Gaussian was not tested in this work by a more thorough analysis, such as the bootstrap method.

\section{MODELING}

The parameters used for the standard binary lens modeling in this paper are the time of closest approach to the barycenter of the lens, $t_{0}$, the Einstein radius crossing time, $t_{\mathrm{E}}$, the impact parameter in units of the Einstein radius, $u_{0}$, the planet-host mass ratio, $q$, the lens separation in the Einstein radius units, $s$, the angle of the source trajectory with respect to the binary axis, $\alpha$, and the angular source radius $\left(\theta_{*}\right)$ normalized by the angular Einstein radius, $\rho \equiv \theta_{*} / \theta_{\mathrm{E}}$. The angular Einstein radius $\theta_{\mathrm{E}}$ is expressed as $\theta_{\mathrm{E}}=\sqrt{\kappa M \pi_{\text {rel }}}$, where $\kappa=4 \mathrm{G} /\left(c^{2} \mathrm{AU}\right)=$
8.14 mas $M_{\odot}^{-1}$, and $M$ is the total mass of the lens system. $\pi_{\text {rel }}$ is the lens-source relative parallax given by $\pi_{\text {rel }}=\pi_{\mathrm{L}}-\pi_{\mathrm{S}}$, where the $\pi_{\mathrm{L}}=\mathrm{AU} / D_{\mathrm{L}}$ and $\pi_{\mathrm{S}}=\mathrm{AU} / D_{\mathrm{S}}$ are the parallax of the lens and that of the source, respectively. $D_{\mathrm{L}}$ is the distance to the lens, and $D_{\mathrm{S}}$ is the distance to the source.

First, we searched the standard model that minimizes $\chi^{2}$ using the above parameters. We used the Markov Chain Monte Carlo (MCMC) method to obtain the $\chi^{2}$ minimum. Light curve calculations were done using a variation of the method of Bennett (2010). The initial parameter sets to search the standard model were used over the wide range, $-5 \leqslant \log q \leqslant 0$ and $-1 \leqslant \log s \leqslant 1$. The total number of initial parameter sets was 858 and all parameters were free parameters. We thereby found that the standard model had $\chi^{2}=6038$ and the parameters are listed in Table 2.

\subsection{Limb Darkening}

The caustic exit is well observed in this event and this implies that finite source effects must be important, because caustic crossings imply singularities in light curves for point sources. We must therefore account for limb darkening when modeling this event. We use a linear limb-darkening model in which the source surface brightness is expressed as

$$
S_{\lambda}(\vartheta)=S_{\lambda}(0)[1-u(1-\cos \vartheta)] .
$$

The parameter $u$ is the limb-darkening coefficient, $S_{\lambda}(0)$ is the central surface brightness of the source, and $\vartheta$ is the angle between the normal to the stellar surface and the line of sight. As discussed in Section 4, the estimated intrinsic source color is $(V-I)_{\mathrm{S}, 0}=0.70$ and its angular radius is $\theta_{*}=0.91 \pm 0.06 \mu$ as. This implies that the source is a mid-late G-type turnoff star. From the source color, we estimate an effective temperature of $T_{\text {eff }} \sim 5690 \mathrm{~K}$ according to González Hernández \& Bonifacio (2009), adopting $\log [\mathrm{M} / \mathrm{H}]=0$. Assuming $\log g=4.0 \mathrm{~cm} \mathrm{~s}^{-2}$, Claret (2000) gives the limb-darkening coefficients for a $T_{\text {eff }} \sim$ $5750 \mathrm{~K}$ star of $u_{I}=0.5251, u_{V}=0.6832$, and $u_{R}=0.6075$, for the $I, V$, and $R$ bands, respectively. We used the average of $I$ - and $R$-band coefficient for the MOA-red wide band and $R$-band coefficient for unfiltered bands.

\subsection{Parallax}

The orbital motion of the Earth during the event implies that the lens does not appear to move at a constant velocity with respect to the source, as seen by Earth-bound observers. This is known as the (orbital) microlensing parallax effect and it can often be detected for events with time scales $t_{\mathrm{E}}>50$ days, like MOA-2010-BLG-328. So, we have included this effect in our modeling. This requires two additional parameters, $\pi_{\mathrm{E}, \mathrm{N}}$ and $\pi_{\mathrm{E}, \mathrm{E}}$, which are the two components of the microlensing parallax vector $\pi_{\mathrm{E}}$ (Gould 2000). The microlensing parallax amplitude is given by $\pi_{\mathrm{E}}=\sqrt{\pi_{\mathrm{E}, \mathrm{N}}^{2}+\pi_{\mathrm{E}, \mathrm{E}}^{2}}$. The amplitude $\pi_{\mathrm{E}}$ is also described as $\pi_{\mathrm{E}}=\pi_{\mathrm{rel}} / \theta_{\mathrm{E}}$. The direction of $\boldsymbol{\pi}_{\mathrm{E}}$ is that of the lens-source relative proper motion at a fixed reference time of HJD $=2455379.0$, which is near the peak of event. If both $\rho$ and $\pi_{\mathrm{E}}$ are measured, one can determine the mass of the lens system:

$$
M=\frac{\theta_{\mathrm{E}}}{\kappa \pi_{\mathrm{E}}}=\frac{\theta_{*}}{\kappa \rho \pi_{\mathrm{E}}},
$$

assuming one also has an estimate of $\theta_{*}$, the angular source radius. Since the source distance, $D_{\mathrm{S}}=\mathrm{AU} / \pi_{\mathrm{S}}$, is approximately 
Table 2

Model Parameters

\begin{tabular}{|c|c|c|c|c|c|c|c|}
\hline Parameters & Standard & Parallax & $\begin{array}{l}\text { Unconstrained } \\
\text { xallarap }\end{array}$ & $\begin{array}{c}\text { Constrained } \\
\text { xallarap }\end{array}$ & Orbital & $\begin{array}{c}\text { Parallax } \\
+ \text { Orbital } \\
\left(u_{0}<0\right)\end{array}$ & $\begin{array}{l}\text { Parallax } \\
+ \text { Orbital } \\
\left(u_{0}>0\right)\end{array}$ \\
\hline $\begin{array}{l}t_{0} \\
\left(\mathrm{HJD}^{\prime}\right)\end{array}$ & $\begin{array}{r}5378.641 \\
0.015 \\
\end{array}$ & $\begin{array}{r}5378.717 \\
0.017 \\
\end{array}$ & $\begin{array}{r}5378.723 \\
0.015 \\
\end{array}$ & $\begin{array}{r}5378.706 \\
0.013 \\
\end{array}$ & $\begin{array}{r}5378.776 \\
0.036 \\
\end{array}$ & $\begin{array}{r}5378.683 \\
0.014 \\
\end{array}$ & $\begin{array}{r}5378.694 \\
0.017 \\
\end{array}$ \\
\hline $\begin{array}{l}t_{\mathrm{E}} \\
\text { (day) }\end{array}$ & $\begin{array}{r}57.2 \\
0.3\end{array}$ & $\begin{array}{r}70.3 \\
0.7\end{array}$ & $\begin{array}{r}62.9 \\
0.3\end{array}$ & $\begin{array}{r}61.8 \\
0.3\end{array}$ & $\begin{array}{r}75.1 \\
0.9\end{array}$ & $\begin{array}{r}62.6 \\
0.6\end{array}$ & $\begin{array}{r}64.2 \\
0.6\end{array}$ \\
\hline$u_{0}$ & $\begin{array}{l}0.0816 \\
0.0005\end{array}$ & $\begin{array}{l}0.0644 \\
0.0007\end{array}$ & $\begin{array}{r}-0.0722 \\
0.0005\end{array}$ & $\begin{array}{r}-0.0741 \\
0.0004\end{array}$ & $\begin{array}{l}0.0596 \\
0.0007\end{array}$ & $\begin{array}{r}-0.0721 \\
0.0008\end{array}$ & $\begin{array}{l}0.0716 \\
0.0007\end{array}$ \\
\hline$q \times 10^{4}$ & $\begin{array}{l}8.16 \\
0.11\end{array}$ & $\begin{array}{l}4.46 \\
0.07\end{array}$ & $\begin{array}{l}5.17 \\
0.08\end{array}$ & $\begin{array}{l}5.16 \\
0.06\end{array}$ & $\begin{array}{r}11.63 \\
0.92\end{array}$ & $\begin{array}{l}2.60 \\
0.53\end{array}$ & $\begin{array}{l}3.68 \\
1.26\end{array}$ \\
\hline$s$ & $\begin{array}{l}1.243 \\
0.001 \\
\end{array}$ & $\begin{array}{l}1.192 \\
0.002 \\
\end{array}$ & $\begin{array}{l}1.216 \\
0.001\end{array}$ & $\begin{array}{l}1.220 \\
0.001 \\
\end{array}$ & $\begin{array}{l}1.310 \\
0.012 \\
\end{array}$ & $\begin{array}{l}1.154 \\
0.016 \\
\end{array}$ & $\begin{array}{l}1.180 \\
0.028 \\
\end{array}$ \\
\hline $\begin{array}{l}\alpha \\
(\mathrm{rad})\end{array}$ & $\begin{array}{l}0.1694 \\
0.0005\end{array}$ & $\begin{array}{l}0.1976 \\
0.0010\end{array}$ & $\begin{array}{r}-0.1740 \\
0.0005\end{array}$ & $\begin{array}{r}-0.2024 \\
0.0004\end{array}$ & $\begin{array}{l}0.1385 \\
0.0081\end{array}$ & $\begin{array}{r}-0.2743 \\
0.0087\end{array}$ & $\begin{array}{l}0.1965 \\
0.0151\end{array}$ \\
\hline$\rho \times 10^{3}$ & $\begin{array}{l}1.91 \\
0.02\end{array}$ & $\begin{array}{l}1.09 \\
0.02\end{array}$ & $\begin{array}{l}1.31 \\
0.02 \\
\end{array}$ & $\begin{array}{l}1.35 \\
0.01\end{array}$ & $\begin{array}{l}1.66 \\
0.06 \\
\end{array}$ & $\begin{array}{l}0.93 \\
0.10\end{array}$ & $\begin{array}{l}1.09 \\
0.17\end{array}$ \\
\hline$\pi_{\mathrm{E}, \mathrm{N}}$ & $\ldots$ & $\begin{array}{l}0.35 \\
0.01\end{array}$ & $\cdots$ & $\cdots$ & $\ldots$ & $\begin{array}{l}1.01 \\
0.06\end{array}$ & $\begin{array}{l}0.72 \\
0.05\end{array}$ \\
\hline$\pi_{\mathrm{E}, \mathrm{E}}$ & $\cdots$ & $\begin{array}{r}-0.13 \\
0.03\end{array}$ & $\ldots$ & $\ldots$ & $\ldots$ & $\begin{array}{r}-0.51 \\
0.04\end{array}$ & $\begin{array}{r}-0.39 \\
0.03\end{array}$ \\
\hline$\xi_{\mathrm{E}, \mathrm{N}}$ & $\cdots$ & $\ldots$ & $\begin{array}{c}-2.58 \\
\ldots\end{array}$ & $\begin{array}{l}0.02 \\
\ldots \\
\end{array}$ & $\ldots$ & $\cdots$ & $\ldots$ \\
\hline$\xi_{\mathrm{E}, \mathrm{E}}$ & $\cdots$ & $\ldots$ & $\begin{array}{c}-1.86 \\
\ldots\end{array}$ & $\begin{array}{l}0.04 \\
\ldots\end{array}$ & $\ldots$ & $\cdots$ & $\ldots$ \\
\hline $\begin{array}{l}\text { R.A. } \xi \\
\text { (deg) }\end{array}$ & $\cdots$ & $\cdots$ & $\begin{array}{c}256.07 \\
\ldots\end{array}$ & $\begin{array}{c}255.77 \\
\ldots\end{array}$ & $\ldots$ & $\cdots$ & $\ldots$ \\
\hline $\begin{array}{l}\text { Decl. } \xi \\
\text { (deg) }\end{array}$ & $\cdots$ & $\ldots$ & $\begin{array}{c}-23.44 \\
\ldots\end{array}$ & $\begin{array}{c}-0.89 \\
\ldots\end{array}$ & $\ldots$ & $\ldots$ & $\ldots$ \\
\hline $\begin{array}{l}P_{\xi} \\
\text { (day) }\end{array}$ & $\cdots$ & $\ldots$ & $\begin{array}{c}475.53 \\
\ldots\end{array}$ & $\begin{array}{c}155.66 \\
\ldots\end{array}$ & $\ldots$ & $\cdots$ & $\ldots$ \\
\hline$\epsilon$ & $\ldots$ & $\ldots$ & $\begin{array}{l}0.17 \\
\ldots\end{array}$ & $\begin{array}{l}0.20 \\
\ldots \\
\end{array}$ & $\ldots$ & $\ldots$ & $\ldots$ \\
\hline $\begin{array}{l}\omega \times 10^{3} \\
\left(\mathrm{rad} \mathrm{day}^{-1}\right)\end{array}$ & $\cdots$ & $\ldots$ & $\ldots$ & $\ldots$ & $\begin{array}{r}-0.93 \\
0.26 \\
\end{array}$ & $\begin{array}{r}-7.39 \\
0.39 \\
\end{array}$ & $\begin{array}{r}-1.39 \\
0.60 \\
\end{array}$ \\
\hline $\begin{array}{l}d s / d t \times 10^{3} \\
\left(\text { day }^{-1}\right)\end{array}$ & $\ldots$ & $\ldots$ & $\ldots$ & $\ldots$ & $\begin{array}{r}-5.67 \\
0.56\end{array}$ & $\begin{array}{l}2.51 \\
0.63\end{array}$ & $\begin{array}{l}1.41 \\
1.16\end{array}$ \\
\hline $\begin{array}{l}\chi^{2} \\
\text { dof }\end{array}$ & $\begin{array}{l}6037.32 \\
5664\end{array}$ & $\begin{array}{l}5684.47 \\
5662\end{array}$ & $\begin{array}{l}5651.69 \\
5658\end{array}$ & $\begin{array}{l}5652.59 \\
5658\end{array}$ & $\begin{array}{l}5716.16 \\
5662\end{array}$ & $\begin{array}{l}5657.75 \\
5660\end{array}$ & $\begin{array}{l}5660.31 \\
5660\end{array}$ \\
\hline
\end{tabular}

Notes. To estimate the errors, the xallarap parameters are fixed at the best values because the xallarap parameters are strongly degenerate. We assumed $M_{\mathrm{S}}=M_{\mathrm{c}}=1 M_{\odot}$ for the constraint in the xallarap model.

known, we can also estimate the lens distance from

$$
D_{\mathrm{L}}=\frac{\mathrm{AU}}{\pi_{\mathrm{E}} \theta_{\mathrm{E}}+\pi_{\mathrm{S}}}=\frac{\mathrm{AU}}{\pi_{\mathrm{E}} \theta_{*} / \rho+\pi_{\mathrm{S}}} .
$$

The parallax model parameters are shown in Table 2 and, as this table indicates, inclusion of the parallax parameters improves $\chi^{2}$ by $\Delta \chi^{2}=353$. When the parallax effect is relatively weak, there is an approximate symmetry in which the lens plane is replaced by its mirror image (i.e., $u_{0} \rightarrow-u_{0}$ and $\alpha \rightarrow-\alpha$ ). However, for this event, this symmetry is broken as the $u_{0}>0$ solution yields a $\chi^{2}$ smaller than the $u_{0}<0$ solution by $\Delta \chi^{2}=78$.

\subsection{Orbital Motion of the Lens Companion}

Another higher order effect that is always present is the orbital motion of the lens system. This causes the shape and position of the caustic curves to change with time. The microlensing signal of the planet can be seen for only $\sim 5$ days, which is much shorter than the likely orbital period of $\sim 8 \mathrm{yr}$, so it is sensible to consider the lowest order components of orbital motion, the two-dimensional relative velocity in the plane of the sky. To lowest order, the orbital motion can be expressed by velocity components in polar coordinates, $\omega$ and $d s / d t$. These are the binary rotation rate and the binary separation velocity (Dong et al. 2009). (Note that this would be a poor choice of variables in cases (e.g., Bennett et al. 2010) where 

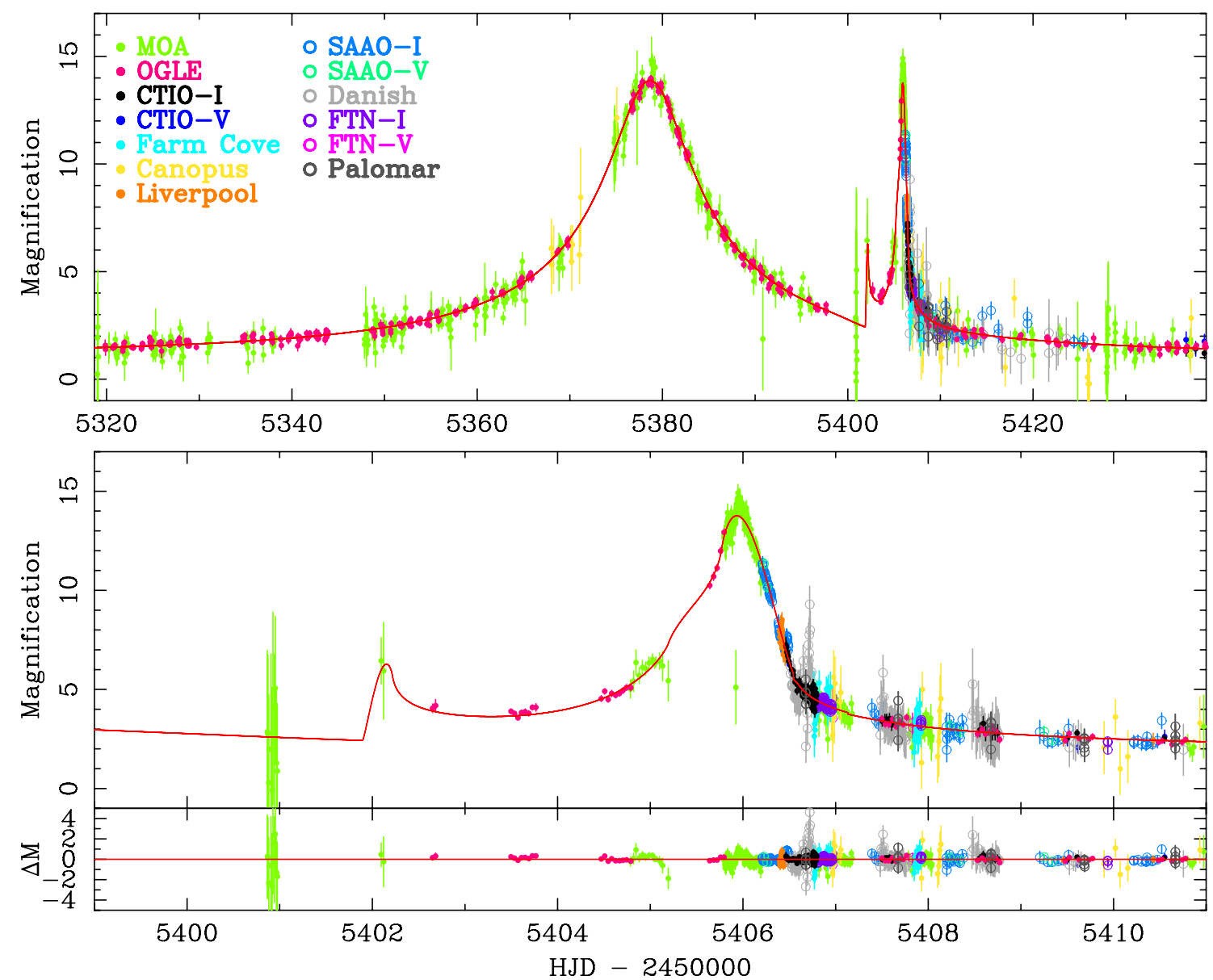

Figure 1. Best parallax plus orbital motion model light curve: the top panel shows the whole light curve and the middle panel shows the anomaly. The bottom panel shows residuals from the model.

the binary acceleration is important, because polar coordinates are not inertial.) When written in the (rotating) lens coordinate system, the source trajectory takes the form

$$
\begin{gathered}
\alpha(t)=\alpha_{0}+\omega\left(t-t_{0}\right), \\
s(t)=s_{0}+d s / d t\left(t-t_{0}\right) .
\end{gathered}
$$

We have conducted fits with both orbital motion alone and with microlensing parallax plus orbital motion and the bestfit parameters for each model are given in Table 2. Figure 1 presents the light curve of the best parallax plus orbital motion model and Figure 2 shows its caustic. This table indicates that the orbital motion-only model improves $\chi^{2}$ by $\Delta \chi^{2}=322$ versus the standard model, which is slightly worse than the $\chi^{2}$ improvement of $\Delta \chi^{2}=353$ for the parallax-only model. The combined parallax and orbital motion model yields a $\chi^{2}$ improvement of $\Delta \chi^{2}=373$. As shown in Table 2, we found that the $u_{0}<0$ model showed smaller $\chi^{2}$ than the $u_{0}>0$ models for the parallax plus orbital motion model, but the difference between $\chi^{2}$ of the $u_{0}<0$ model and that of the $u_{0}>0$ model is small $\left(\Delta \chi^{2} \sim 3\right)$. This is due to the degeneracy of $\pi_{\mathrm{E}, \perp}$ with $\omega$ (Batista et al. 2011; Skowron et al. 2011), where $\pi_{\mathrm{E}, \perp}$ is the component of $\pi_{\mathrm{E}}$ that is perpendicular to the instantaneous direction of the Earth's acceleration. Compared with the $u_{0}>0$ model, the $u_{0}<0$ model is preferred by $\Delta \chi^{2} \sim 3$ but the $u_{0}>0$ model cannot be excluded.

Since both orbital motion and microlensing parallax should exist at some level in every binary microlensing light curve, the parallax plus orbital motion model should be considered

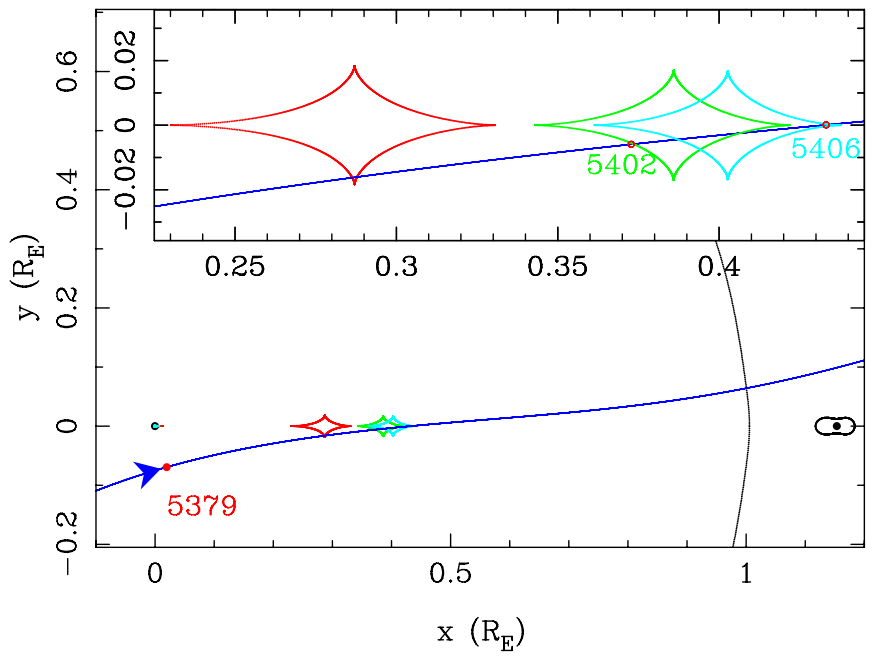

Figure 2. Caustics (red lines) and critical curves (black lines) of the best parallax plus orbital motion model near the peak at $\mathrm{HJD}=2355379$. The source trajectory is shown by the blue lines. The black dot at $(x, y) \sim(1.2,0)$ represents the planet position. The green and cyan lines indicate the caustics when the source enters the caustic at $\mathrm{HJD}=2355402$ and exits at $\mathrm{HJD}=2355406$. The inset shows a closeup of the planetary caustic. The red filled and open circles on the source trajectory are source positions at $\mathrm{HJD}=2355379,2355402$, and 2355406, respectively. The size of the red open circles in the inset indicates the source size.

more realistic than the orbital motion-only model. However, it is important to check that the parameters of the orbital motion models are consistent with the allowed velocities for bound orbits, since the probability of finding planets in unbound orbits 


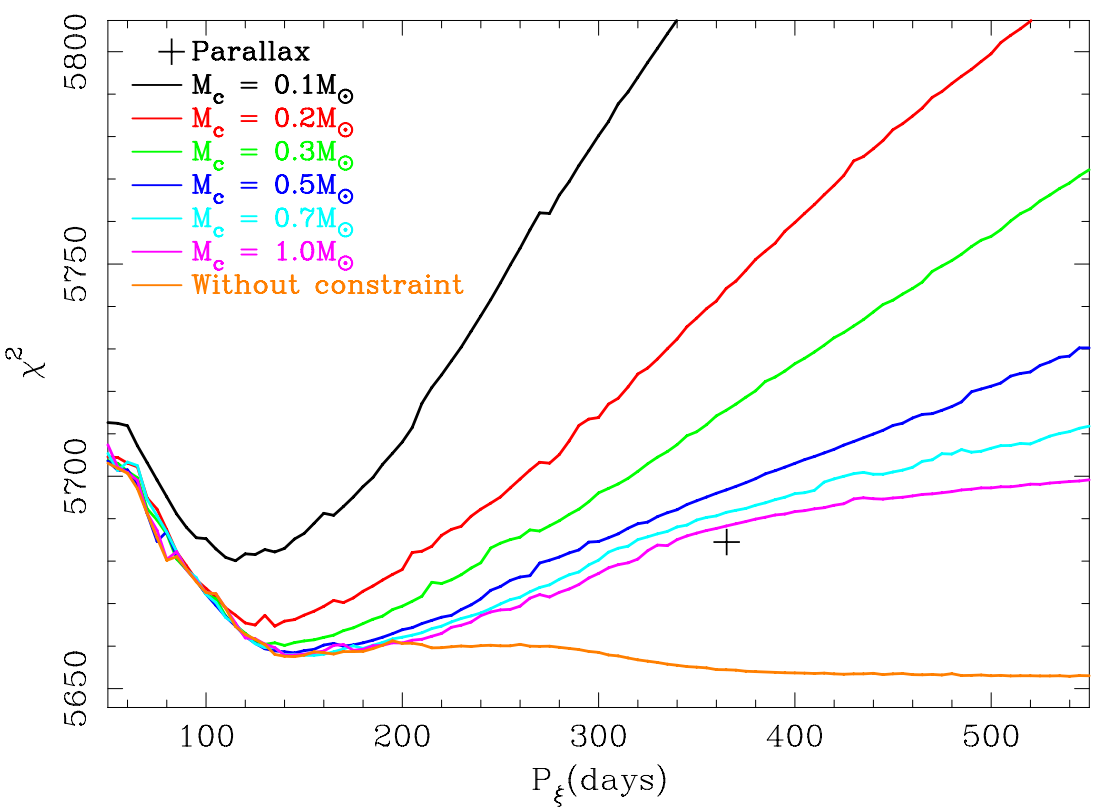

Figure 3. $\chi^{2}$ of the xallarap model as a function of orbital period. The color-coded lines represent the unconstrained model and the constrained model assuming various companion masses, respectively. The "+" indicates the best parallax model (with no orbital motion) for comparison.

is extremely low. So, we would like to be able to compare the transverse kinetic energy, $(\mathrm{KE})_{\perp}=M v_{\mathrm{rel}, \perp}^{2} / 2$, with the potential energy, $(\mathrm{PE})_{\perp}=G M / r_{\perp}$ (Dong et al. 2009). Then, the ratio of kinetic to potential energy can be expressed in terms of observables as

$$
\left(\frac{\mathrm{KE}}{\mathrm{PE}}\right)_{\perp}=\frac{\kappa M_{\odot} \pi_{\mathrm{E}}(|\boldsymbol{\gamma}| \mathrm{yr})^{2} s_{0}^{3}}{8 \pi^{2} \theta_{\mathrm{E}}\left(\pi_{\mathrm{E}}+\pi_{\mathrm{S}} / \theta_{\mathrm{E}}\right)^{3}},
$$

where $\boldsymbol{\gamma}=\left(\gamma_{\|}, \gamma_{\perp}\right)$ consists of $\gamma_{\|}=(d s / d t) / s_{0}$ and $\gamma_{\perp}=\omega$. The parameters of the parallax plus orbital motion model indicate that these ratios are 0.72 and 0.08 for the $u_{0}<0$ and $u_{0}>0$ model, respectively, and this implies that the both models are reasonable.

\subsection{Xallarap}

The xallarap effect is the converse of the parallax effect. It is due to the orbital motion of the source instead of the orbital motion of the observers on the Earth. Xallarap can cause similar light curve distortions to the parallax effect (Poindexter et al. 2005). Unlike parallax and orbital motion, however, there is a good chance that the source will not have a companion with an orbital period in the right range to give a detectable xallarap signal. Only about $10 \%$ of source stars have a companion with orbital parameters that would allow a xallarap solution that could mimic microlensing parallax.

For the xallarap model, the xallarap vector, $\left(\xi_{\mathrm{E}, \mathrm{N}}, \xi_{\mathrm{E}, \mathrm{E}}\right)$, which correspond to $\left(\pi_{\mathrm{E}, \mathrm{N}}, \pi_{\mathrm{E}, \mathrm{E}}\right)$, the direction of observer relative to the source orbital axis, R.A. . and decl. . $_{\xi}$, the orbital period, $P_{\xi}$, the orbital eccentricity, $\epsilon$, and the time of periastron, $t_{\text {peri }}$, are required in addition to the standard binary model.

The xallarap amplitude, $\xi_{\mathrm{E}}$, is expressed with Kepler's third law, as follows:

$$
\xi_{\mathrm{E}}=\frac{a_{\mathrm{s}}}{\hat{r}_{\mathrm{E}}}=\frac{1 \mathrm{AU}}{\hat{r}_{\mathrm{E}}}\left(\frac{M_{\mathrm{c}}}{M_{\odot}}\right)\left(\frac{M_{\odot}}{M_{\mathrm{c}}+M_{\mathrm{S}}} \frac{P_{\xi}}{1 \mathrm{yr}}\right)^{\frac{2}{3}},
$$

where $a_{\mathrm{S}}$ is the semimajor axis of the source orbit and, $M_{\mathrm{S}}$ and $M_{\mathrm{c}}$ are the masses of the source and its companion, respectively.
$\hat{r}_{\mathrm{E}}$ is the Einstein radius projected onto the source plane and is described as follows:

$$
\frac{\hat{r}_{\mathrm{E}}}{\mathrm{AU}}=\theta_{\mathrm{E}} D_{\mathrm{S}}=\frac{\theta_{*}}{\rho} D_{\mathrm{S}} .
$$

Assuming values for the two masses $M_{\mathrm{S}}$ and $M_{\mathrm{c}}$, we can determine $\xi_{\mathrm{E}}$ for a given period $P_{\xi}$ and then we can constrain the $\xi_{\mathrm{E}}$ value in the xallarap model. We conducted the xallarap modeling with constraints and without constraints. For the constrained xallarap model, we assumed $M_{\mathrm{S}}=1 M_{\odot}, D_{\mathrm{S}}=$ $8 \mathrm{kpc}$, and various masses of the companion, $M_{\mathrm{c}}$, from $0.1 M_{\odot}$ to $1 M_{\odot}$. Here, the upper limit of the companion mass is due to the measured blending flux, as shown in Section 5.2.

The parameters obtained for each models are listed in Table 2. At this time, we ignored the orbital motion of the companion of the lens. The best unconstrained and constrained xallarap models (fixed with $M_{\mathrm{c}}=1 M_{\odot}$ ) have nearly identical $\chi^{2}$, i.e., 5652 and 5653, respectively. They produced improved fits with $\Delta \chi^{2} \sim 386$ and 385 compared with the standard model, and $\Delta \chi^{2} \sim 6$ and 5 compared with the parallax plus orbital motion model, respectively. Figure 3 shows the $\chi^{2}$ distribution as a function of $P_{\xi}$. In Figure 3, the orbital eccentricity, $\epsilon$, was fixed as the value for Earth. Only the result with a companion mass of $M_{\mathrm{c}}=0.1 M_{\odot}$ has worse $\chi^{2}$ than the parallax only model for every $P_{\xi}$. Therefore, we estimated the probability of the existence of a companion with $0.2<q<1$ and $80<P_{\xi}<365$, whose $\chi^{2}$ was smaller than the parallax model, and found that the prior probability was about 3\% (Duquennoy \& Mayor 1991).

As discussed earlier in this section, the xallarap signal can mimic the microlensing parallax. If the parallax signal is real, the xallarap parameters should converge on the Earth values. To check whether the parallax is real, we verify the parameters of the unconstrained xallarap model. This is because if the parallax model is correct, the xallarap parameter, $\xi_{\mathrm{E}}$, does not represent a real companion, so it can be anything. Focusing on the period, $P_{\xi}$, as shown in Figure 3, the period is consistent with $P_{\xi}=1 \mathrm{yr}$. Note that the lens orbital motion is ignored in Figure 3. Then, we checked the consistency of the R.A. $\xi$ and decl. $\xi$. To check this, we conducted the xallarap modeling with 


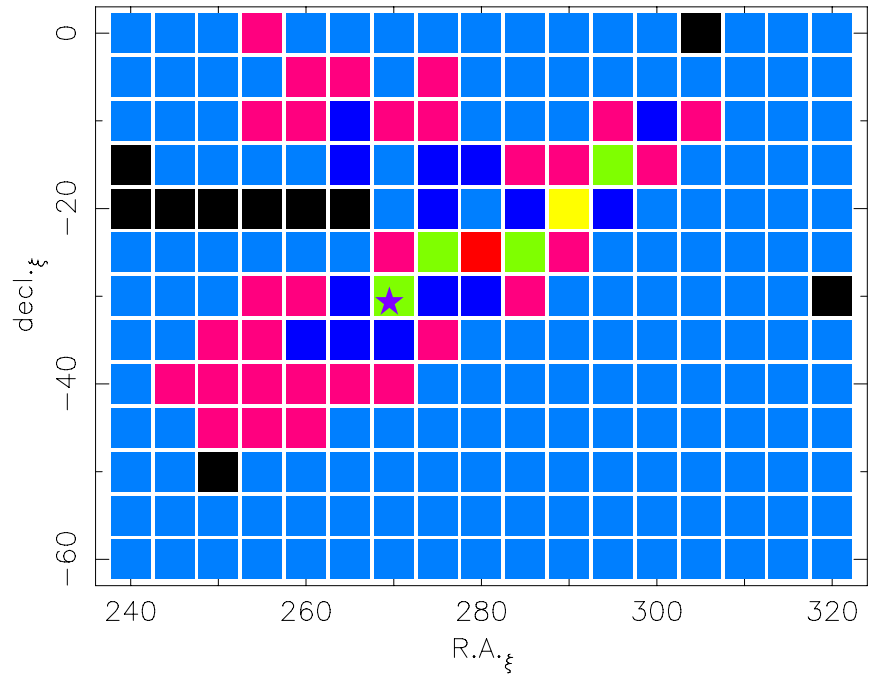

Figure 4. Map of $\chi^{2}$ surface of the unconstrained xallarap model with fixed R.A. $\xi$ and decl. $\xi$ and $u_{0}>0$. The period and eccentricity are fixed at those of the Earth. The orbital motion of the lens is included. The square is colorcoded for solutions with $\Delta \chi^{2}$ within 1 (red), 4 (yellow), 9 (green), 16 (blue), 25 (magenta), 49 (aqua), and above 49 (black). The purple star mark represents the position of the target in the sky plane (R.A. $=269^{\circ}$, decl. $=-31^{\circ}$ ).

R.A. $\xi$ and decl. $\xi$ fixed at a grid of values. During this test, we fixed the eccentricity and period with the Earth's values and included the lens orbital motion, i.e., $\omega, d s / d t$. The $\chi^{2}$ map in the R.A. $\xi$-decl. $\xi$ plane is shown in Figure 4. The best xallarap model has (R.A. $\xi$, decl. $\xi)=(280,-25)$, which is close to the coordinates of the event $\left(\right.$ R.A. $=269^{\circ}$, decl. $\left.=-31^{\circ}\right)$. The $\Delta \chi^{2}$ between the best xallarap model and the nearby coordinates of the event $\left(\right.$ R.A. $=270^{\circ}$, decl. $\left.=-30^{\circ}\right)$ is small $\left(\Delta \chi^{2} \sim 7\right)$. The results of the verification of the consistency of the period and coordinate support that the xallarap parameters are consistent with the Earth parameters. As discussed previously, the prior probability that the source has a companion with the required mass/period parameters is small $(\sim 3 \%)$. Now, even if it did have these parameters, the chance that the orientation of the orbit would mimic that of the Earth's to the degree shown in Figure 4 is only about $0.6 \%$. Combining these two factors yields a prior probability, $B_{\mathrm{x}}$, of only $2 \times 10^{-4}$. On the other hand, we also estimated a prior probability that the lens would be at $0.81 \mathrm{kpc}$ (as derived from the parallax solution), $B_{\mathrm{p}}$, of $2.5 \%$. This is a $2 \sigma$ value of the MCMC chain of the parallax plus orbital motion. Note that the distribution of the error is not a Gaussian. Comparing both prior probabilities, the parallax is preferred by a factor of $B_{\mathrm{p}} / B_{\mathrm{x}}=125$. Moreover, we found that the probability of getting the observed improvement in $\Delta \chi^{2}$ with the xallarap model for additional dof, $P\left(\Delta \chi^{2} ; \mathrm{N}\right)$, was about 0.1 . Even if we consider this probability, the parallax solution is still preferred a factor of $B_{\mathrm{p}} / B_{\mathrm{x}} \times P\left(\Delta \chi^{2} ; \mathrm{N}\right)=12.5$. Nevertheless, the prior probability that the lens would be at 0.81 or $1.24 \mathrm{kpc}$ (as derived from the parallax solution) is also relatively low, so the xallarap model needs to be considered carefully. We therefore estimated the physical parameters of the lens for the parallax plus orbital motion model and for the constrained xallarap model by a Bayesian analysis using $t_{\mathrm{E}}$ and $\theta_{\mathrm{E}}$.

\section{SOURCE STAR PROPERTIES}

\subsection{For Parallax Plus Orbital Motion Model}

We derive the source star angular radius, $\theta_{*}$, in order to obtain the angular Einstein radius, $\theta_{\mathrm{E}}$. The model-independent source

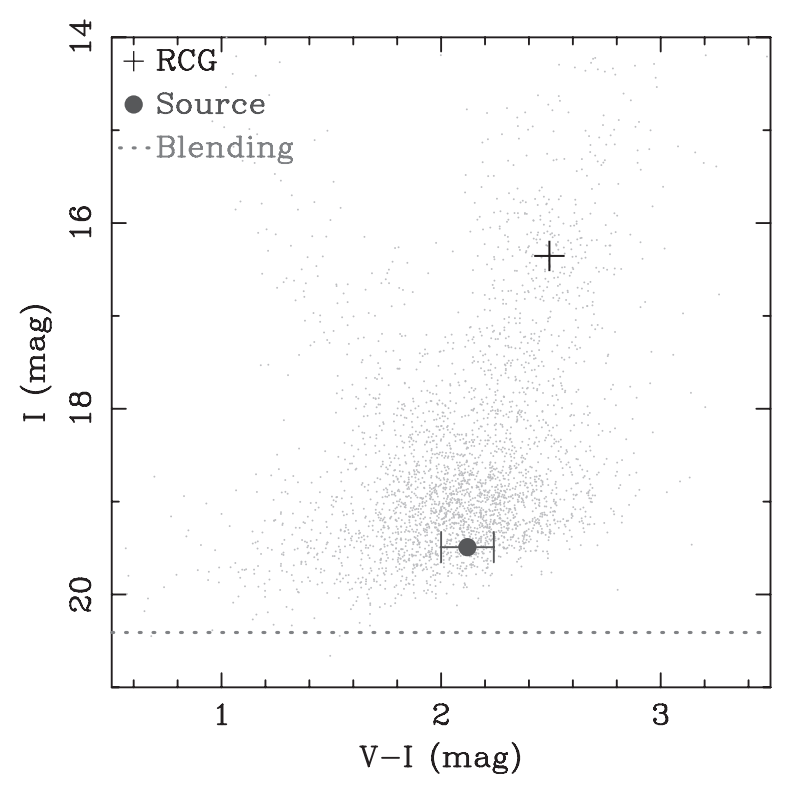

Figure 5. OGLE-III CMD of stars within 1'.5 from the source star of MOA-2010-BLG-328. The filled circle represents the $I$-band magnitude of the source and the horizontal dashed lines indicates the blended light in the best parallax plus orbital motion model. The cross indicates the center of RCGs.

color is derived from $V$ - and $I$-band photometry from CTIO using linear regression and the observed magnitude of the source is determined from OGLE $I$ band data with modeling. However, these are affected by interstellar dust. This means that we need to estimate the intrinsic source color and magnitude. Therefore, we use the red clump giants (RCGs), which are known to be approximate standard candles. We adopt the intrinsic RCG color $(V-I)_{\mathrm{RCG}, 0}=1.06 \pm 0.06$ (Bensby et al. 2011) and the magnitude $M_{I, \mathrm{RCG}, 0}=14.45 \pm 0.04$ (Nataf et al. 2012):

$$
(V-I, I)_{\mathrm{RCG}, 0}=(1.06,14.45) \pm(0.06,0.04) .
$$

We construct two color-magnitude diagrams (CMDs): one is constructed from the OGLE-III catalog and the other is constructed from the instrumental CTIO photometry. The CMD of OGLE-III is used for the calibration of the I-band magnitude and the CMD of CTIO is used for the calibration of the color. Figure 5 shows the CMD constructed from the OGLE-III catalog. From the CMD, the I-band magnitude of the RCG centroid is estimated to be $I_{\mathrm{RCG}, \mathrm{obs}}=16.28$. By comparing the intrinsic RCG and observed one, we find that the offset is $\Delta I_{\mathrm{RCG}}=1.83$. Applying this offset to the observed $I$-band magnitude of the source, $I_{\mathrm{S} \text {, obs }}=19.49\left(u_{0}<0\right.$ model $)$, we obtain the intrinsic $I$-band magnitude of the source, $I_{\mathrm{S}, 0}=$ 17.66. Likewise, we estimate that the instrumental color of the RCG centroid of the CTIO CMD to be $(V-I)_{\mathrm{RCG}, \mathrm{obs}}=0.80$. The color offset of the RCGs between the intrinsic and the observed value is $\Delta(V-I)_{\mathrm{RCG}}=0.26$. Using this offset to calibrate the observed color of the source, $(V-I)_{\mathrm{S} \text {, obs }}=0.44$, we can obtain the intrinsic color of the source: $(V-I)_{\mathrm{S}, 0}=0.70$. Finally, we find the intrinsic $I$-band magnitude and the color of the source to be

$$
(V-I, I)_{\mathrm{S}, 0}=(0.70,17.66) \pm(0.10,0.04)\left(u_{0}<0\right) .
$$

Using color-color relation (Bessell \& Brett 1988), we derive $(V-K, K)_{\mathrm{S}, 0}$ from $(V-I, I)_{\mathrm{S}, 0}$ :

$$
(V-K, K)_{\mathrm{S}, 0}=(1.51,16.89) \pm(0.23,0.26)\left(u_{0}<0\right) .
$$


Then, we apply the relation between $(V-K, K)_{\mathrm{S}, 0}$ and the stellar angular radius (Kervella et al. 2004) and estimate the source star angular radius:

$$
\theta_{*}=0.91 \pm 0.06 \mu \operatorname{as}\left(u_{0}<0\right) .
$$

Adopting same procedure for the $u_{0}>0$ model, we derive $\theta_{*}=0.90 \pm 0.06 \mu \mathrm{as}$. This source star angular radius is consistent with that obtained from the $u_{0}<0$ model. These source star angular radii mean that the source star radius is $1.5 R_{\odot}$ assuming that the source star is located in the Galactic bulge $(\sim 8 \mathrm{kpc})$. The color of the source star indicates that the source star is a $\mathrm{G}$ star, and the estimated source star radius is slightly larger than that of typical $\mathrm{G}$ dwarfs. For this reason, we conclude that the source star is a G subgiant or a turnoff star. From the finite source effect parameter, $\rho$, in the parallax plus orbital motion model, we drive the angular Einstein radius and source-lens relative proper motion, $\mu$, for the $u_{0}<0$ model:

$$
\begin{gathered}
\theta_{\mathrm{E}}=\frac{\theta_{*}}{\rho}=0.98 \pm 0.12 \text { mas, } \\
\mu=\frac{\theta_{\mathrm{E}}}{t_{\mathrm{E}}}=5.71 \pm 0.70 \mathrm{mas} \mathrm{yr}^{-1},
\end{gathered}
$$

and for $u_{0}>0$ model

$$
\begin{gathered}
\theta_{\mathrm{E}}=0.83 \pm 0.14 \text { mas, } \\
\mu=4.72 \pm 0.79 \mathrm{mas} \mathrm{yr}^{-1} . \\
\text { 4.2. For the Constrained Xallarap Model }
\end{gathered}
$$

\subsection{For the Constrained Xallarap Model}

According to the same procedure used for the parallax plus orbital motion model, we also estimated source star properties for the case of the constrained xallarap model. The source color and magnitude are largely similar to those obtained from the parallax plus orbital motion model:

$$
\begin{gathered}
(V-I, I)_{\mathrm{S}, 0}=(0.70,17.63) \pm(0.10,0.04), \\
(V-K, K)_{\mathrm{S}, 0}=(1.51,16.83) \pm(0.23,0.26) .
\end{gathered}
$$

From these source colors and magnitudes, we derived the angular Einstein radius and source-lens relative proper motion:

$$
\begin{gathered}
\theta_{\mathrm{E}}=0.68 \pm 0.04 \text { mas, } \\
\mu=4.03 \pm 0.26 \mathrm{mas} \mathrm{yr}^{-1} .
\end{gathered}
$$

\section{LENS SYSTEM}

\subsection{Parallax Plus Orbital Motion Model}

For determining the mass and distance of lens system, we combine Equations (2), (3), and the microlensing parallax parameter, $\pi_{\mathrm{E}}$, which was derived from the parallax plus orbital motion model. For the $u_{0}<0$ model, Equation (2) yields a host star mass of $M_{\mathrm{h}}=0.11 \pm 0.01 M_{\odot}$ and a planet mass of $M_{\mathrm{p}}=9.2 \pm 2.2 M_{\oplus}$. To determine the distance to the lens system with Equation (3), we need the source distance, $D_{\mathrm{S}}$, which we assume to be $D_{\mathrm{S}}=8.0 \pm 0.3 \mathrm{kpc}$ (Yelda et al. 2010), i.e., $\pi_{\mathrm{S}}=0.125 \pm 0.005 \mathrm{mas}$, and this gives a lens distance of
$D_{\mathrm{L}}=0.81 \pm 0.10 \mathrm{kpc}$. The projected star-planet separation is therefore $r_{\perp}=s D_{\mathrm{L}} \theta_{\mathrm{E}}=0.92 \pm 0.16 \mathrm{AU}$. This implies that the lens is a very nearby red star. The probability distributions of the mass, distance, Einstein radius, and brightnesses of the lens are shown in Figure 6.

The mass of the host star, derived from the parallax plus orbital motion model, indicates that the absolute $J$-, $H$-, and $K$-band magnitudes would be $M_{J}=10.06 \pm 0.29, M_{H}=9.49 \pm 0.27$, and $M_{K}=9.16 \pm 0.25 \mathrm{mag}$, respectively (Kroupa \& Tout 1997). Marshall et al. (2006) calculated the extinction distribution in three dimensions. According to them, the extinction in the $K$-band at $D_{\mathrm{L}}=0.81 \pm 0.10 \mathrm{kpc}$ is $A_{K}=0.05 \pm 0.01$. The Cardelli et al. (1989) extinction law gives infrared extinction ratios of $A_{J}: A_{H}: A_{K}=1: 0.67: 0.40$, which imply that the $J$ - and $H$-band extinctions are $0.13 \pm 0.02$ and $0.09 \pm 0.01$. With these extinctions and the derived distance modulus, the apparent $J, H$, and $K$ magnitudes of the host (and lens) star would be $J_{\mathrm{L}}=19.73 \pm 0.39, H_{\mathrm{L}}=19.13 \pm 0.37$, and $K_{\mathrm{L}}=18.76 \pm 0.36 \mathrm{mag}$, respectively.

For the $u_{0}>0$ model, we find a host star mass of $M_{\mathrm{h}}=$ $0.12 \pm 0.02 M_{\odot}$, a planet mass of $M_{\mathrm{p}}=15.2 \pm 5.9 M_{\oplus}$, and a lens distance of $D_{\mathrm{L}}=1.24 \pm 0.18 \mathrm{kpc}$. The projected star-planet separation is therefore $r_{\perp}=1.21 \pm 0.27 \mathrm{AU}$. The mass of the host star indicates that the absolute $\mathrm{J}$-, $\mathrm{H}$-, and $\mathrm{K}$-band magnitudes would be $M_{J}=9.74 \pm 0.38, M_{H}=9.19 \pm 0.36$, and $M_{K}=8.88 \pm 0.34 \mathrm{mag}$, respectively. The distance to the lens indicates that the extinction in the $J, H$, and $K$ bands is $A_{J}=0.20 \pm 0.03, A_{H}=0.14 \pm 0.02$, and $A_{K}=0.08 \pm 0.01$. With these extinctions and the derived distance modulus, the apparent $J, H$, and $K$ magnitudes of the host (and lens) star would be $J_{\mathrm{L}}=20.40 \pm 0.50, H_{\mathrm{L}}=19.80 \pm 0.48$, and $K_{\mathrm{L}}=19.42 \pm 0.47 \mathrm{mag}$, respectively.

\subsection{Constrained Xallarap Model}

For the xallarap model, we estimate lens properties using a Bayesian analysis. We can obtain only the Einstein angular radius, $\theta_{\mathrm{E}}$, from the finite source effect parameter, $\rho$, in the xallarap model. Consequently, for a Bayesian analysis, we combined Equations (2), (3), and $\theta_{\mathrm{E}}, t_{\mathrm{E}}$ with the Galactic model (Han \& Gould 2003) and mass function.

The mass function is based on Sumi et al. (2011) Table 3S model \#1, but we apply a slight modification. Sumi et al. (2011) assumed that stars that were initially above $1 M_{\odot}$ have evolved into stellar remnants. However, we assume the fraction of stars with masses above $1 M_{\odot}$ by referencing Bensby et al. (2011). Bensby et al. (2011) obtained spectra of 26 microlensed stars and found that 12 stars were old and metal poor and 14 stars were young and metal rich stars. So, we assume that the mass function is constructed both by old, metal-poor stars and young, metal-rich stars equally. From the isochrones of Demarque et al. (2004), we conclude that old, metal-poor stars, the initial masses of which were above $1 M_{\odot}$, have evolved into stellar remnants and the young, metal-rich stars, the initial masses of which were above $1.2 M_{\odot}$, have evolved into stellar remnants. Hence, the mass function has a $50 \%$ fraction of an initial mass function above $1 M_{\odot}$ and has a cutoff at $1.2 M_{\odot}$.

Additionally, we used the I-band blended magnitude as an upper limit. The blended light derived from the modeling is $I_{\mathrm{b}, \mathrm{obs}}=20.49 \pm 0.11$. Even if the lens lies behind all the dust, it cannot be more reddened than the source. Thus we applied the same extinction as the source and found $I_{\mathrm{b}, 0}=18.66 \pm 0.12$. Therefore, we used this blended light as an upper limit in the Bayesian analysis. 

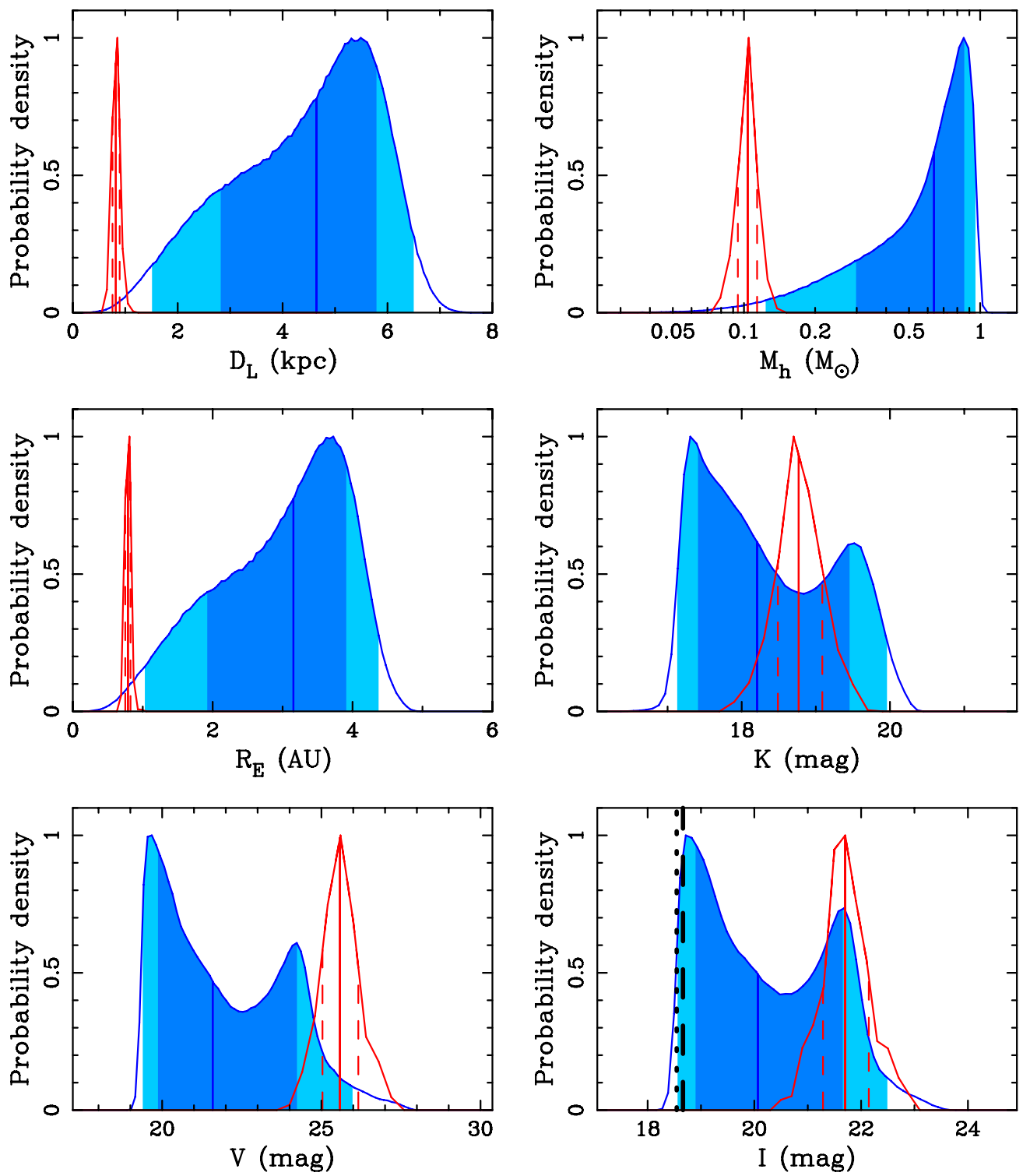

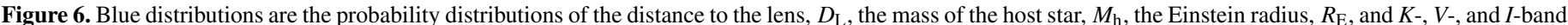

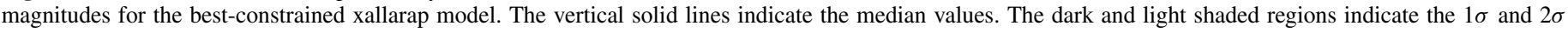

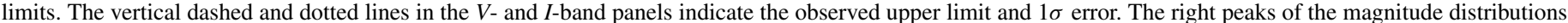

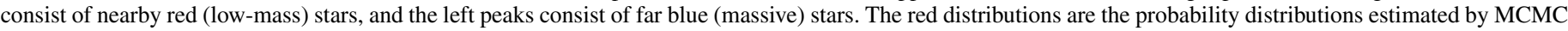
chains of the best parallax plus orbital model for comparison. The vertical solid and dashed lines indicate the median values and the $1 \sigma$ limits.

Figure 6 shows the probability distributions obtained by the Bayesian analysis. From the analysis, we find that the host star is a $\mathrm{K}$ dwarf with a mass $M_{\mathrm{h}}=0.64_{-0.34}^{+0.22} M_{\odot}$ and a distance $D_{\mathrm{L}}=4.6_{-1.8}^{+1.1} \mathrm{kpc}$. The planet has a Saturn-like mass, $M_{\mathrm{p}}=109_{-58}^{+38} M_{\oplus}=1.15_{-0.61}^{+0.40} M_{\text {Saturn. The Einstein radius }}$ is $R_{\mathrm{E}}=3.2_{-1.2}^{+0.8}$, implying that the projected separation is $r_{\perp}=3.8_{-1.5}^{+0.9} \mathrm{AU}$. The physical three-dimensional separation is $a=4.6_{-1.7}^{+1.9} \mathrm{AU}$, estimated by putting a planetary orbit at a random inclination and phase (Gould \& Loeb 1992).

The Bayesian analysis also yields the $J_{-}, \mathrm{H}$-, and $\mathrm{K}$-band magnitudes of the host star, which are $J_{\mathrm{L} 0}=18.95_{-0.99}^{+1.32}, H_{\mathrm{L} 0}=$ $18.37_{-0.85}^{+1.33}$, and $K_{\mathrm{L} 0}=18.21_{-0.80}^{+1.25}$ mag, respectively, without extinction. In Figure 6, the distributions of the magnitude have two peaks. The right peak consists of nearby red (low-mass) stars and the left peak consists of far blue (massive) stars. The distance to the lens indicates extinctions of $A_{J}=0.72_{-0.25}^{+0.01}, A_{H}=$
$0.49_{-0.17}^{+0.01}$, and $A_{K}=0.29_{-0.10}^{+0.01}$. According to these estimates, the apparent magnitudes of the host (and lens) star should be $J_{\mathrm{L}}=19.67_{-1.02}^{+1.32}, H_{\mathrm{L}}=18.86_{-0.87}^{+1.33}$, and $K_{\mathrm{L}}=18.50_{-0.81}^{+1.25} \mathrm{mag}$.

\section{DISCUSSION AND CONCLUSIONS}

We report the analysis of the planetary microlensing event MOA-2010-BLG-328. The higher order effect improved the $\chi^{2}$ and the constrained xallarap model yielded the smallest $\chi^{2}$ value. However, the difference of the $\chi^{2}$ between the constrained xallarap and the parallax plus lens orbital motion model is small $\left(\Delta \chi^{2}=5\right)$ and the xallarap model has a high probability of mimicking the parallax for this event. We found that the mass ratio and separation are $(2.60 \pm 0.53) \times 10^{-4}$ and $1.154 \pm 0.016$ Einstein radii for the best $u_{0}<0$ parallax plus orbital model, $(3.68 \pm 1.26) \times 10^{-4}$ and $1.180 \pm 0.028$ Einstein radii for the best $u_{0}>0$ parallax plus orbital model, and $(5.17 \pm 0.08) \times 10^{-4}$ and 
$1.216 \pm 0.001$ Einstein radii for the best-constrained xallarap model.

Using the parallax parameter $\pi_{\mathrm{E}}$, we can determine the physical parameters of the lens uniquely. In the case of the $u_{0}<0$ model, the mass of the host star and distance to the lens are derived to be $M=0.11 \pm 0.01 M_{\odot}$ and $D_{\mathrm{L}}=$ $0.81 \pm 0.10 \mathrm{kpc}$. The mass of the planet is $M_{\mathrm{p}}=9.2 \pm 2.2 M_{\oplus}$ and the projected separation is $r_{\perp}=0.92 \pm 0.16 \mathrm{AU}$. On the other hand, in the case of the $u_{0}>0$ model, the mass of the host star and distance to the lens are derived to be $M=0.12 \pm 0.02 M_{\odot}$ and $D_{\mathrm{L}}=1.24 \pm 0.18 \mathrm{kpc}$. The mass of the planet is $M_{\mathrm{p}}=15.2 \pm 5.9 M_{\oplus}$ and the projected separation is $r_{\perp}=1.21 \pm 0.27 \mathrm{AU}$. These imply that the lens system consists of a low-mass star and a cold sub-Neptune planet.

We also estimated the probability distributions of physical parameters of the lens system using Bayesian analysis with $t_{\mathrm{E}}$ and $\theta_{\mathrm{E}}$, which were derived from the constrained xallarap model. The Bayesian analysis yields that the host star is a K dwarf with a mass of $M_{\mathrm{h}}=0.64_{-0.34}^{+0.22} M_{\odot}$ at $D_{\mathrm{L}}=4.6_{-1.8}^{+1.1} \mathrm{kpc}$ and the planet mass is $M_{\mathrm{p}}=109_{-58}^{+38} M_{\oplus}=1.15_{-0.61}^{+0.40} M_{\text {Saturn }}$ with a projected separation of $r_{\perp}=3.8_{-1.5}^{+0.9}$ AU.

As discussed in Section 3.4, the unconstrained xallarap model shows a smaller $\chi^{2}$ value than that of the parallax plus orbital motion model, but only by $\Delta \chi^{2}=5$ for two more dof. While formally significant, this difference could also be caused by rather modest systematic errors and we found that the parallax is preferred rather than the xallarap. High angular resolution follow-up observations with the Hubble Space Telescope (HST) or adaptive optics (AO) can be used to confirm if the parallax plus orbital motion model is correct observationally. If the AO or HST observations are conducted, they should resolve stars unrelated to the source and lens stars that are blended with the lens and source stars in seeing-limited, ground-based images. This should allow the brightness of the combined lens and source stars to be determined. Because we know the source brightness from the models, we can get the brightness of the lens by subtracting the brightness of the source from the brightness measured by the $H S T$ or AO observations. If the lens brightnesses derived from the parallax plus orbital motion model differ vastly from those derived from the xallarap model, we can confirm if the parallax plus orbital motion model is correct. As shown in Section 5, the probability distributions of lens brightnesses of xallarap model have two peaks. The brighter one consists of blue turnoff dwarf stars with of about solar mass at the far side of the disk. The fainter one consists of late $\mathrm{M}$ dwarfs with $M \sim 0.2-0.4 M_{\odot}$ in the closer disk at $2-4 \mathrm{kpc}$. On the other hand, the lens of the parallax plus orbital motion model is redder and closer than the stars in this fainter peak. So, these models can be distinguished by brightness measurements in multiple bands. Furthermore, if the observations with $H S T$ were to be conducted after a few years, when the lens and source have separated far enough for their relative positions to be determined (Bennett et al. 2007), we can determine the direction of the lens-source motion. Then, we can confirm if the parallax plus orbital motion model is correct by comparing the observed direction of the lens-source motion with the parallax plus orbital motion prediction.

OGLE has started their EWS and issued 1744 microlens alerts in 2012. Additionally, the Wise Observatory in Israel began survey observations with a $1 \mathrm{~m}$ telescope equipped with a $1 \operatorname{deg}^{2}$ FOV camera. The Korean Microlensing Telescope Network, which is a network using three $1.6 \mathrm{~m}$ telescopes with $4.0 \mathrm{deg}^{2}$ CCD cameras, will provide continuous coverage of microlensing events. These facilities will enable us to obtain well-covered data for most microlensing events and find more planetary events. If we could observe the planetary anomalies without the need for follow-up observations, statistical analysis of the exoplanet distribution by microlensing would become easier, because we would not need to consider the effect of the follow-up observations on the detection efficiency. With more robust statistics, we could approach an understanding of exoplanets from various directions, such as the dependence of the mass of the host star.

We acknowledge the following sources of support: the MOA project is supported by the Grant-in-Aid for Scientific Research (JSP19340058, JSPS20340052, and JSPS22403003) and the Global COE Program of Nagoya University "Quest for Fundamental Principles in the Universe" from JSPS and MEXT of Japan. The OGLE project has received funding from the European Research Council under the European Community's Seventh Framework Programme (FP7/2007-2013)/ERC grant agreement no. 246678 to A.U. A.G. acknowledges support from NSF AST-1103471. B.S.G., A.G., L.-W.H., and R.W.P. acknowledge support from NASA grant NNX12AB99G. J.C.Y. was supported by a National Science Foundation Graduate Research Fellowship under Grant No. 2009068160. B.S. and J.v.S. are also supported by National Science Foundation Graduate Research Fellowships. C.H. was supported by the Creative Research Initiative Program (2009-0081561) of the National Research Foundation of Korea. Work by S.D. was performed under contract with the California Institute of Technology (Caltech) funded by NASA through the Sagan Fellowship Program. This work is based in part on data collected by MiNDSTEp with the Danish $1.54 \mathrm{~m}$ telescope at the ESO La Silla Observatory. The Danish $1.54 \mathrm{~m}$ telescope is operated based on a grant from the Danish Natural Science Foundation (FNU). The MiNDSTEp monitoring campaign is powered by ARTEMiS (Automated Terrestrial Exoplanet Microlensing Search; Dominik et al. 2008). M.H. acknowledges support by the German Research Foundation (DFG). D.R. (boursier FRIA) and J.S. acknowledge support from the Communauté Française de Belgique-Actions de Recherche Concertées-Académie Universitaire WallonieEurope. K.A., D.M.B., M.D., K.H., M.H., C.L., C.S., R.A.S., and Y.T. are thankful to Qatar National Research Fund (QNRF), a member of the Qatar Foundation, for support by grant NPRP 09-476-1-078. T.C.H. gratefully acknowledges financial support from the Korea Research Council for Fundamental Science and Technology (KRCF) through the Young Research Scientist Fellowship Program. T.C.H. acknowledges financial support from KASI (Korea Astronomy and Space Science Institute) grant number 2013-9-400-00. C.U.L. acknowledges financial support from KASI grant number 2012-1-410-02. K.H. is supported by a Royal Society Leverhulme Trust Senior Research Fellowship.

\section{REFERENCES}

Albrow, M. D., Horne, K., Bramich, D. M., et al. 2009, MNRAS, 397, 2099 Bachelet, E., Shin, I.-G., Han, C., et al. 2012, ApJ, 754, 73

Batista, V., Gould, A., Dieters, S., et al. 2011, A\&A, 529, A102 Beaulieu, J.-P., Bennett, D. P., Fouqué, P., et al. 2006, Natur, 439, 437 Bennett, D. P. 2010, ApJ, 716, 1408

Bennett, D. P., Anderson, J., \& Gaudi, B.S. 2007, ApJ, 660, 781

Bennett, D. P., Bond, I. A., Udalski, A., et al. 2008, ApJ, 684, 663 Bennett, D. P., \& Rhie, S. H. 1996, ApJ, 472, 660

Bennett, D. P., Rhie, S. H., Nikolaev, S., et al. 2010, ApJ, 713, 837

Bensby, T., Adén, D., Meléndez, J., et al. 2011, A\&A, 533, A134 Bessell, M. S., \& Brett, J. M. 1988, PASP, 100, 1134

Bond, I. A., Abe, F., Dodd, R. J., et al. 2001, MNRAS, 327, 868

Bond, I. A., Udalski, A., Jaroszyński, M., et al. 2004, ApJL, 606, L155 
Bramich, D. M. 2008, MNRAS, 386, L77

Cardelli, J. A., Clayton, G. C., \& Mathis, J. S. 1989, ApJ, 345, 245

Cassan, A., Kubas, D., Beaulieu, J.-P., et al. 2012, Natur, 481, 167

Claret, A. 2000, A\&A, 363, 1081

Cumming, A., Butler, R. P., Marcy, G. W., et al. 2008, PASP, 120, 531

Demarque, P., Woo, J.-H., Kim, Y.-C., \& Yi, S. K. 2004, ApJS, 155, 667

Dominik, M., Horne, K., Allan, A., et al. 2008, AN, 329, 248

Dominik, M., Jørgensen, U. G., Rattenbury, N. J., et al. 2010, AN, 331, 671

Dong, S., Gould, A., Udalski, A., et al. 2009, ApJ, 695, 970

Duquennoy, A., \& Mayor, M. 1991, A\&A, 248, 485

Gaudi, B. S., Bennett, D. P., Udalski, A., et al. 2008, Sci, 319, 927

González Hernández, J. I., \& Bonifacio, P. 2009, A\&A, 497, 497

Gould, A. 2000, ApJ, 542, 785

Gould, A., Dong, S., Gaudi, B. S., et al. 2010, ApJ, 720, 1073

Gould, A., \& Loeb, A. 1992, ApJ, 396, 104

Gould, A., Udalski, A., An, D., et al. 2006, ApJL, 644, L37

Griest, K., \& Safizadeh, N. 1998, ApJ, 500, 37

Han, C., \& Gould, A. 2003, ApJ, 592, 172

Horne, K., Snodgrass, C., \& Tsapras, Y. 2009, MNRAS, 396, 2087

Howard, A. W., Marcy, G. W., Bryson, S. T., et al. 2012, ApJS, 201, 15

Kennedy, G. M., \& Kenyon, S. J. 2008, ApJ, 673, 502

Kervella, P., Thévenin, F., Di Folco, E., \& Ségransan, D. 2004, A\&A, 426, 297
Kroupa, P., \& Tout, C. A. 1997, MNRAS, 287, 402

Kubas, D., Beaulieu, J. P., Bennett, D. P., et al. 2012, A\&A, 540, 78

Lovis, C., \& Fischer, D. 2011, in Exoplanets, ed. S. Seager (Tucson, AZ: Univ. Arizona Press), 27

Marshall, D. J., Robin, A. C., Reylé, C., Schultheis, M., \& Picaud, S. 2006, A\&A, 453, 635

Nataf, D. M., Gould, A., Fouqué, P., et al. 2012, arXiv:1208.1263

Poindexter, S., Afonso, C., Bennett, D. P., et al. 2005, ApJ, 633, 914

Sako, T., Sekiguchi, T., Sasaki, M., et al. 2008, ExA, 22, 51

Schechter, P. L., Mateo, M., \& Saha, A. 1993, PASP, 105, 1342

Skowron, J., Udalski, A., Gould, A., et al. 2011, ApJ, 738, 87

Smith, M. C., Mao, S., \& Paczyński, B. 2003, MNRAS, 339, 925

Sumi, T., Abe, F., Bond, I. A., et al. 2003, ApJ, 591, 204

Sumi, T., Bennett, D. P., Bond, I. A., et al. 2010, ApJ, 710, 1641

Sumi, T., Kamiya, K., Bennett, D. P., et al. 2011, Natur, 473, 349

Tsapras, Y., Street, R., Horne, K., et al. 2009, AN, 330, 4

Udalski, A. 2003, AcA, 53, 291

Udalski, A., Jaroszyński, M., Paczyński, B., et al. 2005, ApJL, 628, L109

Winn, J. N. 2011, in Exoplanets, ed. S. Seager (Tucson, AZ: Univ. Arizona Press), 55

Wittenmyer, R. A., Tinney, C. G., O’Toole, S. J., et al. 2011, ApJ, 727, 102

Wozniak, P. R. 2000, AcA, 50, 421

Yelda, S., Lu, J. R., Ghez, A. M., et al. 2010, ApJ, 725, 331 